\title{
Implementación de un curso de habilidades quirúrgicas y procedimientos básicos en simulación para residentes de traumatología
}

\author{
Implementation of a course on surgical skills and basic \\ procedures in simulation for traumatology residents \\ Catalina Vidal,* Aron Kuroiwa-Rivero, ${ }^{*, \ddagger}$ Pablo Besa, ${ }^{*}$ \\ Sebastián Irarrázaval,* Paula Pino,* Jorge Vergara,* Luis Irribarra*
}

Palabras clave:
Simulación,
ortopedia, educación,
residentes.
Keywords:
Simulation,
orthopedics,

education, residents.

\section{* Departamento \\ de Ortopedia y \\ Traumatología, \\ Pontificia Universidad \\ Católica de Chile. \\ ¥ Servicio de \\ Traumatología, \\ Complejo Asistencial \\ Sótero del Río. \\ Santiago, Chile.}

Recibido: 25/05/2021

Aceptado: 27/07/2021

doi: $10.35366 / 101428$

\section{RESUMEN}

Introducción: La adquisición de competencias técnicas en un programa de especialidad de traumatología es un proceso complejo y requiere entrenamiento. Objetivo: Describir la implementación de un curso de habilidades quirúrgicas y procedimientos básicos en simulación para residentes de traumatología. Evaluar de manera secundaria los cambios del desempeño de los residentes. Material y métodos: Se incluyeron 10 residentes de traumatología de primer año. Se organizaron seis estaciones de evaluación y entrenamiento: lavado de manos y pabellón; inmovilización con yeso; sutura; cortes con sierra; brocado y artrocentesis. Se realizaron videos para la demostración de las técnicas, así como pautas de observación directa para las evaluaciones. Al finalizar, se realizó una encuesta para evaluar la satisfacción de los participantes. Resultados: Diez residentes de primer año completaron el curso. En cinco de las seis estaciones hubo mejoras significativas en el desempeño posterior al entrenamiento. Cien por ciento de los participantes evaluó el curso como bueno o excelente; refirieron haber aumentado su nivel de conocimientos y recomendaron su implementación en el futuro. Conclusión: La implementación de un curso de habilidades quirúrgicas y procedimientos básicos al inicio de la residencia tuvo una evaluación positiva de los residentes y mejoró su desempeño en estas competencias.

\section{ABSTRACT}

Introduction: The acquisition of technical competencies in an orthopedic specialty program is a complex process that requires training. Objective: To describe the implementation of a course of surgical skills and basic simulation procedures for orthopedic residents. Secondly, to evaluate changes on resident performance. Material and methods: Ten first-year orthopedic residents were included. Six stations were organized: hand washing and operating room; cast application; suture; oscillating saw cuts; drilling and knee arthrocentesis. Videos were recorded to demonstrate the techniques and direct observation guidelines were used for evaluations. At the end, a survey was conducted to assess the satisfaction of the participants. Results: Ten first-year residents completed the course. There were significant improvements in performance in five of the six stations. All the participants evaluated the course as good or excellent; reported having increased their level of knowledge and recommended its implementation in the future. Conclusion: The implementation of a course of surgical skills and basic procedures at the beginning of the residency had a positive evaluation of the residents and improved their performance.

\section{INTRODUCCIÓN}

L os programas de las especialidades médicas deben lograr, como parte de sus objetivos, la adquisición de competencias técnicas por parte de los residentes. Esto puede ser un proceso complejo y que requiere un entrena- miento específico. ${ }^{1,2}$ Las altas exigencias en actividades clínicas, extensos horarios tanto de residentes como especialistas ${ }^{3-5}$ y las preocupaciones por la seguridad de los pacientes en contextos de formación ${ }^{6}$ han disminuido la exposición necesaria para la adquisición de estas competencias.

Citar como: Vidal C, Kuroiwa-Rivero A, Besa P, Irarrázaval S, Pino P, Vergara J, et al. Implementación de un curso de habilidades quirúrgicas y procedimientos básicos en simulación para residentes de traumatología. Rev Latinoam Simul Clin. 2021; 3 (2): 55-62. https://dx.doi.org/10.35366/101428 
Frente a esto, la simulación ha permitido un aumento del aprendizaje debido a que propicia la práctica deliberada en escenarios estructurados y seguros, además de introducir nuevas habilidades que históricamente se han adquirido en las salas del hospital o en el pabellón, y al mismo tiempo evaluar, corregir y mejorar. ${ }^{7}$ La ACGME (Accreditation Council for Graduate Medical Education's Residency Review Committee) ha reconocido que el entrenamiento basado en simulación tiene un rol importante en el desarrollo de habilidades técnicas y ha sugerido que todos los programas de residencia tengan alguna forma de entrenamiento simulado. ${ }^{8}$ Diferentes laboratorios de habilidades quirúrgicas desarrollados en distintas instituciones han tenido resultados positivos en la formación de especialistas. ${ }^{9,10}$

En el caso de Ortopedia y Traumatología, el rol de la simulación como forma de aprendizaje tiene cada vez mayor evidencia, ${ }^{11-14}$ siendo particularmente relevante para los residentes jóvenes quienes muchas veces no se sienten cómodos en el escenario real o tienen poco acceso a estos escenarios para desarrollar sus competencias. ${ }^{15,16}$ Con el propósito de disminuir los riesgos dentro del pabellón y asegurar un aprendizaje de competencias prácticas, nacen los programas intensivos de adquisición de habilidades básicas.

El objetivo principal de este estudio es describir la implementación de un curso de simulación de habilidades quirúrgicas y procedimientos básicos para residentes de Ortopedia y Traumatología al inicio de su formación. El objetivo secundario es evaluar cambios en el desempeño de los residentes después del curso de simulación.

\section{MATERIAL Y MÉTODOS}

Se diseñó un curso de tres días en un centro de simulación universitario para los residentes de primer año de Ortopedia y Traumatología durante el año 2020, el cual es parte del programa de inducción obligatoria de los residentes que se realiza en la primera semana al inicio de su residencia.

Para el curso se planificaron seis estaciones de entrenamiento: lavado de manos y pabellón; inmovilización con yeso; suturas; cortes con sierra; brocado; y artrocentesis (Figura 1). Se seleccionaron estos procedimientos debido a la importancia de su adquisición durante el desarrollo del programa.

El curso práctico se realizó en cuatro fases:

1. Estudio individual de cada residente: una semana antes del inicio del curso, se enviaron videos instruccionales a los residentes con la demostración de las técnicas y procedimientos, además de material de estudio relacionado a ello.

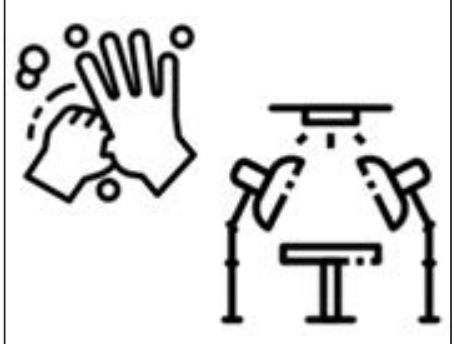

Lavado de manos y pabellón

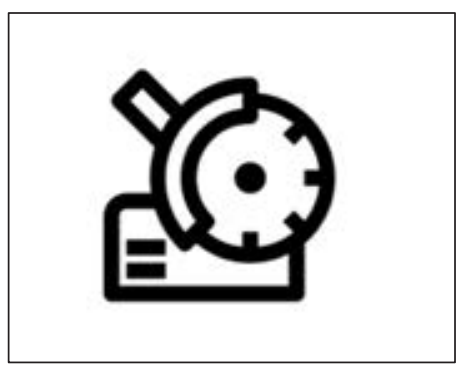

Cortes con sierra

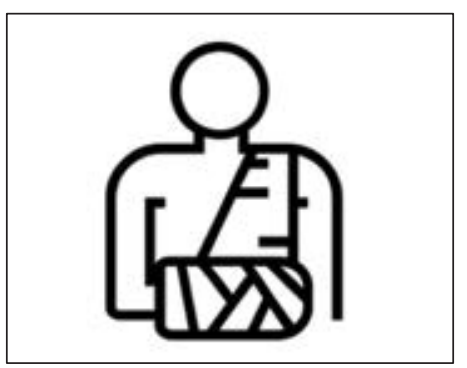

Inmovilización con yeso

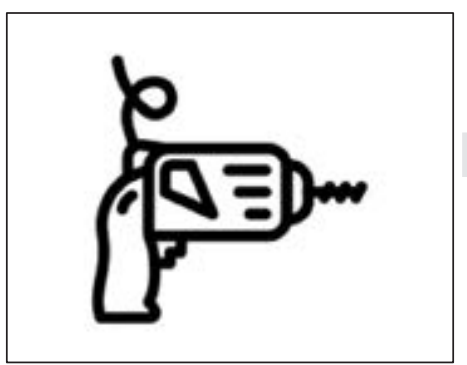

Brocado

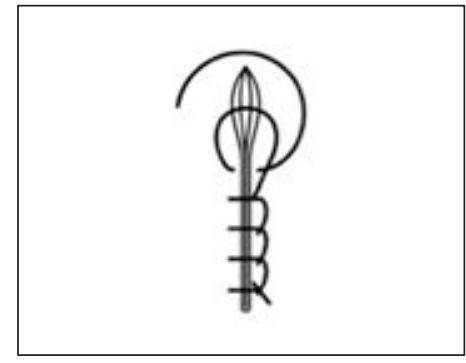

Suturas

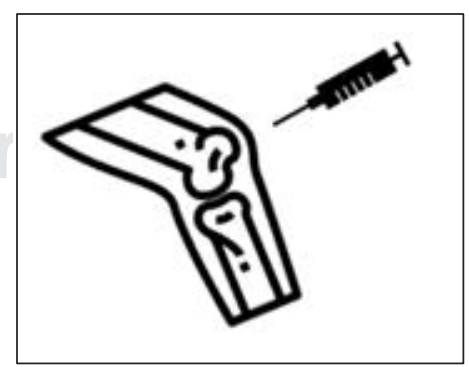

Artrocentesis

Figura 1: Estaciones diseñadas para curso de entrenamiento de residentes. 
Tabla 1: Objetivos y desarrollo de cada estación de entrenamiento.

\begin{tabular}{|c|c|c|c|}
\hline Estación & Objetivo & Modelo de simulación & Desarrollo \\
\hline $\begin{array}{l}\text { Lavado de manos y } \\
\text { pabellón }\end{array}$ & $\begin{array}{l}\text { Conocer conceptos } \\
\text { de antisepsia y su } \\
\text { aplicación en el pabellón } \\
\text { quirúrgico }\end{array}$ & Fantoma & $\begin{array}{l}\text { Cada residente realiza en parejas un lavado de manos con } \\
\text { jabón, luego con crema. Después se reviste con guantes y bata } \\
\text { estéril para pincelar y armar campos estériles desechables }\end{array}$ \\
\hline Inmovilización con yeso & $\begin{array}{l}\text { Dominar la confección } \\
\text { de la valva de yeso en } \\
\text { tenaza de azúcar }\end{array}$ & Paciente simulado & $\begin{array}{l}\text { El residente selecciona, mide y corta los materiales a utilizar. } \\
\text { Luego posiciona al paciente y realiza una valva de yeso en } \\
\text { tenaza de azúcar. Se evalúa también el retiro correcto del } \\
\text { yeso al finalizar }\end{array}$ \\
\hline Suturas & $\begin{array}{l}\text { Dominar las técnicas } \\
\text { básicas de sutura }\end{array}$ & Kit de sutura silicona & $\begin{array}{l}\text { El residente elige el instrumental adecuado para realizar dos } \\
\text { suturas de un plano cada una: } 8 \mathrm{~cm} \text { de intradérmica y } 8 \mathrm{~cm} \\
\text { de puntos simples separados }\end{array}$ \\
\hline Cortes con sierra & $\begin{array}{l}\text { Introducir al alumno en } \\
\text { el uso sierras oscilantes }\end{array}$ & Trozos de madera & $\begin{array}{l}\text { El residente arma el motor para la sierra oscilante y se } \\
\text { protege con guantes y lentes de seguridad. Elige el tamaño } \\
\text { correcto de sierra para las cuñas dibujadas en trozos de } \\
\text { madera. Por último, realiza los cortes y deja el motor } \\
\text { bloqueado sobre la mesa }\end{array}$ \\
\hline Brocado & $\begin{array}{l}\text { Introducir al alumno en } \\
\text { el uso del motor }\end{array}$ & $\begin{array}{l}\text { Trozos de madera y } \\
\text { tubos de PVC } \\
\text { (policloruro de vinilo) }\end{array}$ & $\begin{array}{l}\text { El residente arma el motor para broca y se protege con guantes } \\
\text { y lentes de seguridad. Luego, muestra las funciones en el mate- } \\
\text { rial que simula un hueso, practicando control de profundidad, } \\
\text { triangulación y palpación. Realiza nuevamente cada ejercicio, } \\
\text { dejando finalmente el motor bloqueado sobre la mesa }\end{array}$ \\
\hline Artrocentesis & $\begin{array}{l}\text { Dominar la realización } \\
\text { de una artrocentesis de } \\
\text { rodilla }\end{array}$ & $\begin{array}{l}\text { Paciente simulado }+ \\
\text { modelo de rodilla } \\
\text { SawBones }{ }^{\circledR}\end{array}$ & $\begin{array}{l}\text { El residente se enfrenta a un caso clínico con paciente simulado } \\
\text { con el cual debe interactuar. Luego, prepara los elementos para } \\
\text { la punción a partir de un carro de enfermería básico. Finalmente, } \\
\text { realiza la punción en un modelo o de rodilla SawBones }{ }^{\circledR}\end{array}$ \\
\hline
\end{tabular}

2. Evaluación previa al entrenamiento: el primer día se evaluó el desempeño de los participantes con pautas designadas en todas las estaciones. Éstas correspondían a pautas de evaluación validadas o creadas por el equipo investigador (puntuación para procedimiento específico-SRS).

3. Entrenamiento de habilidades y procedimientos: se permitió la práctica de los residentes, luego de la ejemplificación por parte del tutor y demostración de los errores más comunes de cada estación. Durante el entrenamiento, los residentes recibían retroalimentación inmediata respecto a su desempeño.

4. Evaluación posterior al entrenamiento: al finalizar el tiempo de entrenamiento, se evaluó nuevamente a los residentes con las pautas designadas, para analizar los cambios en el rendimiento de cada estación.

Al finalizar el curso, se aplicó una encuesta de satisfacción a los residentes que participaron, con el objetivo de realizar una evaluación global y valorar de forma específica la estructura, contenido y docentes de cada estación.

Para analizar los resultados obtenidos, se utilizó estadística descriptiva reportando frecuencias absolutas y relativas. Para comparar puntaje de desempeño en las estaciones previo y posterior al entrenamiento, se utilizó la prueba estadística Wilcoxon signed-rank test, reportando medianas y rangos. Se realizó a través del software estadístico Stata v.14. Este proyecto fue aprobado por el comité ético científico de investigación (ID: 200821001).

\section{RESULTADOS}

Se implementaron las seis estaciones de entrenamiento en simulación en septiembre del año 2020, cada una contó con un objetivo de aprendizaje e instrucciones para su desarrollo (Tabla 1). Este curso se planifica para la primera semana de inicio de la residencia; sin embargo, debido a la pandemia COVID-19, debió retrasarse cuatro 
meses, hasta que estuvieron las condiciones para permitir la movilidad y aforo necesario.

Cuatro meses previos al inicio, se hizo un recuento de los materiales, insumos y recursos que se utilizarían para cada estación. Todo esto se revisó con el docente especialista encargado de cada estación, y se discutió con el equipo para resolver dudas. La semana anterior se instalaron las estaciones en el Centro de Simulación de la universidad, organizando un cronograma para que cada residente tuviera un tiempo asignado para las evaluaciones y para el entrenamiento tanto guiado como libre. Se enviaron los videos instruccionales y las pautas de evaluación.

Diez residentes de primer año completaron el curso de entrenamiento en simulación (Figura 2). La mediana de edad fue 27 años (mín. 25-máx. 32) y $90 \%$ (9) eran hombres. Participaron además 10 traumatólogos especialistas y siete residentes de segundo y tercer año para realizar las evaluaciones antes y después del entrenamiento y apoyar en la retroalimentación.

Se realizó la evaluación y el entrenamiento en la totalidad de las estaciones y hubo mejoras significativas en cinco de las seis estaciones: inmovilización con yeso, suturas, cortes con sierra, brocado y artrocentesis $(p<0.05)$ (Tabla 2). La mediana del tiempo de entrenamiento libre fue de 135 minutos (mín. 30-máx. 240).

En la encuesta de satisfacción del curso, $100 \%$ de los residentes lo evaluó como bueno o excelente. La mayor proporción refirió que su nivel de conocimientos previo al inicio del curso era deficiente o regular, y al finalizar el curso la totalidad refirió que era bueno o excelente (Figura 3). Usando un puntaje de 1 a 7 , las estaciones mejor evaluadas fueron inmovilización con yeso y artrocentesis (Figura 4). Por último, un 100\% recomendó el curso a otros residentes de Ortopedia y Traumatología.

\section{DISCUSIÓN}

Se desarrolló un curso de habilidades quirúrgicas y procedimientos básicos en residentes de traumatología, con seis estaciones de evaluación y entrenamiento.

Se observó una mejora significativa en el rendimiento de residentes en cinco de las seis estaciones al terminar el curso. Diferentes revisiones sistemáticas y meta-análisis han demostrado, en cursos de entrenamiento quirúrgico, mejoras en outcomes de conocimientos, habilidades técnicas, desempeño y confianza. ${ }^{17,18}$ También han sido reportados entrenamientos en el área de la cirugía, ${ }^{19,20}$ cirugía general ${ }^{21,22}$ y cardiología, ${ }^{23}$ entre otros. Un estudio realizado en el año 2020 demostró que un curso de entrenamiento en alumnos de medicina de habilidades en sutura, colocación de catéter central, intubación, entre otros mejora el desempeño y la confianza de los estudiantes para desarrollar todas las tareas. ${ }^{24}$ Esto
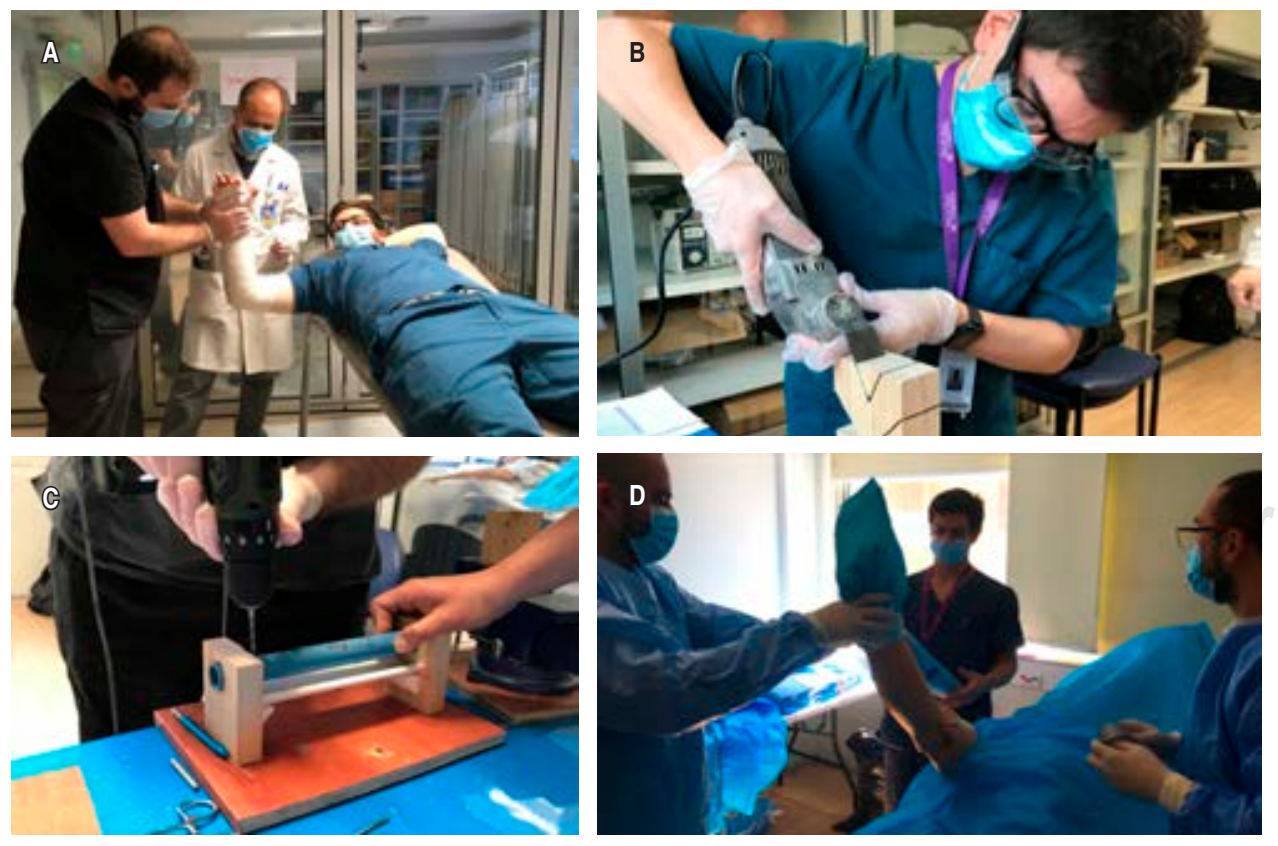

Figura 2:

Desarrollo de las estaciones de simulación: A) Estación de inmovilización con yeso.

B) Estación de cortes con sierra.

C) Estación de brocado. D) Estación de lavado de manos y pabellón. 
Tabla 2: Desempeño en las estaciones previo y posterior al entrenamiento.

\begin{tabular}{|c|c|c|c|}
\hline Estación & $\begin{array}{l}\text { Preentrenamiento } \\
\text { Mediana (mín-máx) }\end{array}$ & $\begin{array}{l}\text { Postentrenamiento } \\
\text { Mediana (mín-máx) }\end{array}$ & $\mathrm{p}$ \\
\hline Lavado de manos y pabellón & $15(13-18)$ & $17.5(14-18)$ & 0.08 \\
\hline Inmovilización con yeso & $10.5(6-16)$ & $16(13-18)$ & $0.007 *$ \\
\hline \multicolumn{4}{|l|}{ Suturas } \\
\hline Simple & $6(4-8)$ & $8(6-10)$ & $0.007 *$ \\
\hline Intradérmica & $8(0-13)$ & $13.5(10-15)$ & $0.006^{*}$ \\
\hline Cortes con sierra & $10(9-13)$ & $11.5(11-14)$ & $0.034 *$ \\
\hline \multicolumn{4}{|l|}{ Brocado } \\
\hline Pauta 1 & $6(4-7)$ & $8(7-9)$ & $0.005^{*}$ \\
\hline OSATS & $21(16-25)$ & $27.5(25-28)$ & $0.005^{*}$ \\
\hline Artrocentesis & $6(4-7)$ & $6.5(6-8)$ & $0.01 *$ \\
\hline
\end{tabular}

permitiría no sólo adquirir destrezas mínimas para enfrentarse al escenario real, sino también usar ese espacio para adquirir destrezas más complejas con mayor seguridad.

En el área de la Ortopedia y Traumatología, los primeros estudios con reportes de cursos intensivos de habilidades quirúrgicas o Surgical Boot Camp se realizaron en Canadá, ${ }^{12,25-27}$ desarrollados con tiempos variables (desde una semana hasta un mes) y con residentes en distintos niveles de formación, tienen mucho en común con este estudio. Se desarrollaron de forma similar las estaciones de suturas, inmovilización con yeso y cortes con sierra, con diferencias en los modelos de simulación utilizados o las tecnologías implementadas. ${ }^{12}$

Para la evaluación del desempeño en las estaciones, utilizamos pautas validadas en aquéllas en las cuales existía publicación en la literatura (brocado, artrocentesis y sutura). ${ }^{28-31}$ En las demás se debió crear un checklist en escala Likert para evaluar el cumplimiento de hitos importantes. Es necesario desarrollar y validar pautas de evaluación para medir correctamente el desempeño de los residentes en cada estación.

La estación de lavado de manos y pabellón fue la única que no demostró mejoras significativas, observándose altos puntajes desde el inicio del curso. Es probable que esta tarea se lleve a cabo con más frecuencia desde los cursos clínicos y el internado, por lo tanto, podría ser una habilidad mejor desarrollada. Además, al desarrollar el curso luego de cuatro meses del inicio de la residencia, esta habilidad se practicó en mayor proporción que las demás.

Por otro lado, las estaciones mejor evaluadas fueron inmovilización con yeso y artrocentesis. En ambas el modelo de simulación incluyó pacientes simulados, logrando un mayor acercamiento al escenario real. Además, requerían mayor tiempo de desarrollo y, por lo tanto, mayor tiempo de retroalimentación. Estudios que han desarrollado sus escenarios de simulación con pacientes simulados tienen el objetivo de mejorar las habilidades no técnicas o comunicati-

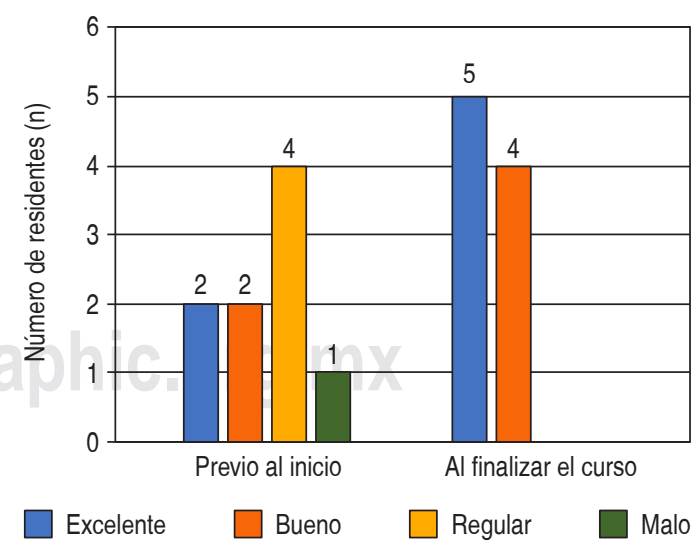

Figura 3: Evaluación subjetiva del nivel de conocimiento por parte de los residentes previo al inicio y al finalizar el curso de entrenamiento. 


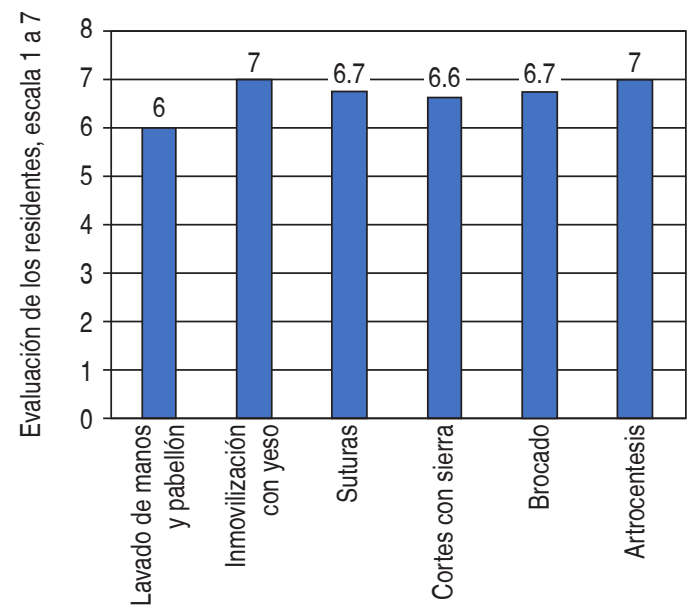

Figura 4: Evaluación de las estaciones por parte de los residentes al finalizar el curso de entrenamiento, en una escala de 1 a 7.

vas. ${ }^{32-35}$ En nuestro caso, el objetivo principal es el de desarrollo de habilidades técnicas, por lo que parece adecuado hacer un equilibrio entre ambos tipos de escenarios de simulación.

Las limitaciones de este proyecto fueron los pocos días para el desarrollo del curso debido a la complejidad de organización. Aproximadamente se necesitaron cuatro meses de planificación para la búsqueda de infraestructura, tiempo y recursos humanos, y se logró obtener una jornada exclusiva de tres días para tener tiempos protegidos por parte de los residentes y especialistas.

Por otro lado, el grupo de participantes fue pequeño, lo que tiene las limitaciones inherentes a calcular resultados en una muestra no representativa de una población. Sin embargo, estaría en concordancia con la mayoría de los estudios publicados en el área. ${ }^{20,36-39}$

Una de las principales fortalezas fue el proceso de evaluación y retroalimentación de los residentes, esencial para lograr los objetivos del curso. En nuestro caso, la participación de residentes de segundo y tercer año facilitó la entrega de retroalimentación basada en una experiencia más cercana, fortaleciendo el aprendizaje tanto de los residentes más grandes como de los novatos, lo que permitió involucrar a los residentes en el proceso educativo y desarrollar una visión profunda y reflexiva respecto al desempeño. ${ }^{40-42}$

\section{CONCLUSIONES}

La implementación de un curso de habilidades quirúrgicas y procedimientos básicos al inicio de la residencia tuvo una evaluación positiva y mejoró el desempeño de los residentes de primer año de Ortopedia y Traumatología.

Futuros estudios pueden indagar en la mantención en el tiempo de las habilidades en cursos de simulación y en la validación de las estaciones de forma independiente junto con la capacidad de transferencia al escenario real.

\section{AGRADECIMIENTOS}

Agradecimientos al Centro de Simulación y Cirugía Experimental de la Pontificia Universidad Católica de Chile.

Agradecimiento a los académicos y residentes que participaron en la organización y desarrollo de este curso de simulación.

\section{REFERENCIAS}

1. Klingensmith ME, Brunt LM. Focused surgical skills training for senior medical students and interns. Surg Clin North Am. 2010; 90 (3): 505-518.

2. lobst WF, Sherbino J, Cate OT, Richardson DL, Dath D, Swing SR, et al. Competency-based medical education in postgraduate medical education. Med Teach. 2010; 32 (8): 651-656.

3. Picarella EA, Simmons JD, Borman KR, Replogle WH, Mitchell ME. "Do one, teach one" the new paradigm in general surgery residency training. J Surg Educ. 2011; 68 (2): 126-129.

4. Jamal MH, Doi SAR, Rousseau M, Edwards M, Rao C, Barendregt JJ, et al. Systematic review and metaanalysis of the effect of North American working hours restrictions on mortality and morbidity in surgical patients. Br J Surg. 2012; 99 (3): 336-344.

5. Antiel RM, Reed DA, Van Arendonk KJ, Wightman SC, Hall DE, Porterfield JR, et al. Effects of duty hour restrictions on core competencies, education, quality of life, and burnout among general surgery interns. JAMA Surg. 2013; 148 (5): 448-455.

6. Britt LD, Sachdeva AK, Healy GB, Whalen TV, Blair PG. Resident duty hours in surgery for ensuring patient safety, providing optimum resident education and training, and promoting resident well-being: a response from the American College of Surgeons to the Report of the Institute of Medicine, "Resident Duty Hours: Enhancing Sleep, Supervision, and Safety". Surgery. 2009; 146 (3): 398-409.

7. Parent RJ, Plerhoples TA, Long EE, Zimmer DM, Teshome M, Mohr CJ, et al. Early, intermediate, and late effects of a surgical skills "boot camp" on an objective structured assessment of technical skills: a randomized controlled study. J Am Coll Surg. 2010; 210 (6): 984-989.

8. Accreditation Council for Graduate Medical Education [Internet]. [Cited 2021 May 3]. Available in: https:// dl.acgme.org/learn/course/the-program-directorsguide-to-the-common-program-requirementsresidency-ebook/interactive-handbook/e-book 
9. Aggarwal R, Moorthy K, Darzi A. Laparoscopic skills training and assessment. Br J Surg. 2004; 91 (12): 1549-1558.

10. Scheeres DE, Mellinger JD, Brasser BA, Davis AT. Animate advanced laparoscopic courses improve resident operative performance. Am J Surg. 2004; 188 (2): 157-160.

11. Howells NR, Gill HS, Carr AJ, Price AJ, Rees JL. Transferring simulated arthroscopic skills to the operating theatre: a randomised blinded study. J Bone Joint Surg Br. 2008; 90 (4): 494-499.

12. Atesok K, Mabrey JD, Jazrawi LM, Egol KA. Surgical simulation in orthopaedic skills training. J Am Acad Orthop Surg. 2012; 20 (7): 410-422.

13. Vaughan N, Dubey VN, Wainwright TW, Middleton RG. Does virtual-reality training on orthopaedic simulators improve performance in the operating room? In: Proceedings of the 2015 Science and Information Conference, SAI 2015 [Internet]. IEEE; 2015 [cited 2021 May 19]. pp. 51-54. Available in: https://research.tees.ac.uk/en/publications/doesvirtual-reality-training-on-orthopaedic-simulatorsimprove-p

14. Ledermann G, Rodrigo A, Besa P, Irarrázaval S. Orthopaedic residents' transfer of knee arthroscopic abilities from the simulator to the operating room. J Am Acad Orthop Surg. 2020; 28 (5): 194-199.

15. McClusky DA, Smith CD. Design and development of a surgical skills simulation curriculum. World J Surg. 2008; 32 (2): 171-181.

16. Reznick RK, MacRae H. Teaching surgical skills-changes in the wind. N Engl J Med. 2006; 355 (25): 2664-2669.

17. Blackmore C, Austin J, Lopushinsky SR, Donnon T. Effects of postgraduate medical education "boot camps" on clinical skills, knowledge, and confidence: a meta-analysis. J Grad Med Educ. 2014; 6 (4): 643-652.

18. Neylan CJ, Nelson EF, Dumon KR, Morris JB, Williams NN, Dempsey DT, et al. Medical school surgical boot camps: a systematic review. J Surg Educ. 2017; 74 (3): 384-389.

19. Antonoff MB, D'Cunha J. PGY-1 surgery preparatory course design: identification of key curricular components. J Surg Educ. 2011; 68 (6): 478-484.

20. Okusanya OT, Kornfield ZN, Reinke CE, Morris JB, Sarani B, Williams NN, et al. The effect and durability of a pregraduation boot CAMP on the confidence of senior medical student entering surgical residencies. J Surg Educ. 2012; 69 (4): 536-543.

21. Peyre SE, Peyre CG, Sullivan ME, Towfigh S. A surgical skills elective can improve student confidence prior to internship. J Surg Res. 2006; 133 (1): 11-15.

22. Todd SR, Fahy BN, Paukert J, Johnson ML, Bass BL. Surgical intern survival skills curriculum as an intern: does it help? Am J Surg. 2011; 202 (6): 713-719.

23. Fann JI, Calhoon JH, Carpenter AJ, Merrill WH, Brown JW, Poston RS, et al. Simulation in coronary artery anastomosis early in cardiothoracic surgical residency training: The Boot Camp experience. J Thorac Cardiovasc Surg. 2010; 139 (5): 1275-1281.

24. Bevilacqua LA, Simon J, Rutigliano D, Sorrento J, Wackett A, Chandran L, et al. Surgical boot camp for fourth-year medical students: impact on objective skills and subjective confidence. Surgery. 2020; 167 (2): 298-301.

25. Sonnadara RR, Van Vliet A, Safir O, Alman B, Ferguson P, Kraemer W, et al. Orthopedic boot camp: examining the effectiveness of an intensive surgical skills course. Surgery. 2011; 149 (6): 745-749.

26. Sonnadara RR, Garbedian S, Safir O, Nousiainen M, Alman B, Ferguson P, et al. Orthopaedic boot camp II: examining the retention rates of an intensive surgical skills course. Surgery. 2012; 151 (6): 803-807.

27. Sonnadara RR, Garbedian S, Safir O, Mui C, Mironova $\mathrm{P}$, Nousiainen $\mathrm{M}$, et al. Toronto orthopaedic boot camp III: examining the efficacy of student-regulated learning during an intensive, laboratory-based surgical skills course. Surgery. 2013; 154 (1): 29-33.

28. Cohen R, Reznick RK, Taylor BR, Provan J, Rothma A. Reliability and validity of the objective structured clinical examination in assessing surgical residents. Am J Surg. 1990; 160 (3): 302-305.

29. Hatala R, Cook DA, Brydges R, Hawkins R. Constructing a validity argument for the objective structured assessment of technical skills (OSATS): a systematic review of validity evidence. Adv Health Sci Educ. 2015; 20 (5): 1149-1175.

30. Denadai R, Saad-Hossne R, Oshiiwa M, Bastos EM. Training on synthetic ethylene-vinyl acetate bench model allows novice medical students to acquire suture skills. Acta Cir Bras. 2012; 27 (3): 271-278.

31. Chipman JG, Schmitz CC. Using objective structured assessment of technical skills to evaluate a basic skills simulation curriculum for first-year surgical residents. J Am Coll Surg. 2009; 209 (3): 364-370.e2.

32. Gorniewicz J, Floyd M, Krishnan K, Bishop TW, Tudiver F, Lang F. Breaking bad news to patients with cancer: a randomized control trial of a brief communication skills training module incorporating the stories and preferences of actual patients. Patient Educ Couns. 2017; 100 (4): 655-666.

33. Kowalski C, Sathanandan S. The use of simulation to develop advanced communication skills relevant to psychiatry. BMJ Simul Technol Enhanc Learn. 2015; 1 (1): 29-32.

34. Liénard A, Merckaert I, Libert Y, Bragard I, Delvaux N, Etienne AM et al. Is it possible to improve residents breaking bad news skills? A randomised study assessing the efficacy of a communication skills training program. Br J Cancer. 2010; 103 (2): 171-177.

35. Yuan YY, Scott S, Van Horn N, Oke O, Okada P. Objective evaluation of a simulation course for residents in the pediatric emergency medicine department: breaking bad news. Cureus [Internet]. 2019 [cited 2021 May 6]. Available in: https://www. cureus.com/articles/17154-objective-evaluation-ofa-simulation-course-for-residents-in-the-pediatricemergency-medicine-department-breaking-bad-news

36. Boehler ML, Rogers DA, Schwind CJ, Fortune J, Ketchum J, Dunnington G. A senior elective designed to prepare medical students for surgical residency. Am J Surg. 2004; 187 (6): 695-697.

37. Esterl RM, Henzi DL, Cohn SM. Senior medical student "Boot Camp": can result in increased self-confidence before starting surgery internships. Curr Surg. 2006; 63 (4): 264-268. 
38. Naylor RA, Hollett LA, Castellvi A, Valentine RJ, Scott DJ. Preparing medical students to enter surgery residencies. Am J Surg. 2010; 199 (1): 105-109.

39. Tocco N, Brunsvold M, Kabbani L, Lin J, Stansfield B, Mueller D, et al. Innovation in internship preparation: an operative anatomy course increases senior medical students' knowledge and confidence. Am J Surg. 2013; 206 (2): 269-279.

40. Preece R, Dickinson EC, Sherif M, Ibrahim Y, Ninan AS, Aildasani L, et al. Peer-assisted teaching of basic surgical skills. Med Educ Online. 2015; 20 (1): 27579.

41. Lockspeiser TM, O'Sullivan P, Teherani A, Muller J. Understanding the experience of being taught by peers: the value of social and cognitive congruence. Adv Health Sci Educ. 2008; 13 (3): 361-372.

42. Steele DJ, Medder JD, Turner P. A comparison of learning outcomes and attitudes in student- versus faculty-led problem-based learning: an experimental study. Med Educ. 2000; 34 (1): 23-29.

\author{
Correspondencia: \\ Aron Kuroiwa-Rivero \\ E-mail: kr.aron@gmail.com
}

